\title{
Alteration of Gut Microbiota in type 2 Diabetes Complicated with Cholelithiasis Patients
}

Jiajia Chen

Peking University Shougang Hospital https://orcid.org/0000-0002-5363-1389

\section{Linlin Yan}

Peking University Shougang Hospital

\section{Xingfan $\mathrm{Ma}$}

Peking University Shougang Hospital

\section{Ping Yuan}

Peking University Shougang Hospital

\section{Fan Zhao}

Peking University Shougang Hospital

\section{Zihan Han}

Beijing Cancer Hospital

Jingshan Liu

Peking University Shougang Hospital

\section{Wenbo Wang}

Peking University Shougang Hospital

\section{Donghai Zhou}

Peking University Shougang Hospital

\section{Hongyu Zhao}

Peking University Shougang Hospital

\section{Nan Feng}

Peking University Shougang Hospital

\section{Dandan Huang}

Peking University Shougang Hospital

\section{Shoukui Hu}

Peking University Shougang Hospital

Jin Gu ( $\nabla$ zlguj@bjmu.edu.cn )

https://orcid.org/0000-0002-9650-1963

\section{Research}


Keywords: Gut microbiota, Type 2 diabetes, Cholelithiasis, Type 2 diabetes complicated with cholelithiasis, 16S rRNA gene sequencing

Posted Date: August 5th, 2020

DOI: https://doi.org/10.21203/rs.3.rs-52504/v1

License: (c) (i) This work is licensed under a Creative Commons Attribution 4.0 International License. Read Full License 
1 Alteration of gut microbiota in type 2 diabetes complicated with 2 cholelithiasis patients

3

4 Jiajia Chen ${ }^{1, \dagger}$, Linlin Yan ${ }^{1, \uparrow}$, Xingfan $\mathrm{Ma}^{1, \uparrow}$, Ping Yuan ${ }^{1}$, Fan Zhao ${ }^{1}$, Zihan Han $^{2}$,

5 Jingshan $\mathrm{Liu}^{1}$, Wenbo Wang ${ }^{1}$, Donghai Zhou ${ }^{1}$, Hongyu Zhao ${ }^{1}$, Nan Feng ${ }^{1}$, Dandan

6 Huang ${ }^{1}$, Shoukui $\mathrm{Hu}^{1, *}$ and Jin $\mathrm{Gu}^{1,2,3, *}$

7

$8{ }^{1}$ Peking University Shougang Hospital, Beijing 100144, China;

$9{ }^{2}$ Key Laboratory of Carcinogenesis and Translational Research, Peking

10 University Cancer Hospital \& Institute, Beijing 100142, China;

$11{ }^{3}$ Peking-Tsinghua Center for Life Sciences, Beijing 100084, China

$12 \dagger$ These authors contributed equally to this work.

13

14 * Correspondence: zlguj@bjmu.edu.cn (Jin Gu), shoukuihu@163.com (Shoukui Hu). 


\section{ABSTRACT}

Background: Epidemiological studies showed that diabetes patients are more prone to developing cholelithiasis. Although composition of gut microbiota in type 2 diabetes or cholelithiasis have been studied respectively, the underlying role of gut microbiota in developing from diabetes to cholelithiasis remains unclear. By 16S rRNA gene sequencing, the gut microbial composition of 33 healthy subjects, 53 type 2 diabetes, 31 cholelithiasis and 32 type 2 diabetes complicated with cholelithiasis patients were studied.

Results: Microbial diversity significantly decreased in type 2 diabetes complicated with cholelithiasis patients. In type 2 diabetes patients, phylum Proteobacteria, class Gammaproteobacteria and order Lactobacillales were significantly increased. In cholelithiasis patients, phylum Bacteroidetes, class Bacteroidia, order Bacteroidales, family Bacteroidaceae and genus Bacteroides were significantly increased. There were also significant increases of phylum Proteobacteria, class Gammaproteobacteria, order Lactobacillales, family Lactobacillaceae and genus Lactobacillus in type 2 diabetes complicated with cholelithiasis patients accompanied by elevated serum triglyceride and total bile acids.

Conclusions: The results show similar but more intricate gut microbiota dysbiosis in type 2 diabetes complicated with cholelithiasis compared with type 2 diabetes, which might partially explain the mechanism of type 2 diabetes as the risk factor of cholelithiasis from the perspective of gut microbiota.

Keywords: Gut microbiota; Type 2 diabetes; Cholelithiasis; Type 2 diabetes complicated with cholelithiasis; 16S rRNA gene sequencing

\section{INTRODUCTION}

Type 2 diabetes and cholelithiasis are both common diseases with a high prevalence. The incidence of type 2 diabetes is about $10.8 \%$ in the United States ${ }^{[1]}$. Cholelithiasis is one of the most common gastrointestinal disease in the Europe and United States, with an incidence between $10-15 \%$ among adults ${ }^{[2,3]}$. In China, the 
prevalence of type 2 diabetes is $9.7 \%$ and adult cholelithiasis is $8-10 \%{ }^{[4]}$. Obesity, insulin resistance and abnormal metabolism are risk factors of type 2 diabetes, epidemiologic studies suggest that which are also risk factors of cholelithiasis ${ }^{[5]}$. As a metabolic system disease, type 2 diabetes may cause chronic damage of multiple tissues and organs. Gallstone formation was reported mainly related to the abnormal metabolism of cholesterol and bile acids ${ }^{[6]}$. Several studies have indicated that diabetes patients are at an increased risk of developing cholelithiasis ${ }^{[7,8]}$. An animal study has shown that insulin resistance directly promotes the formation of gallstones ${ }^{[9]}$. However, the underlying mechanism that diabetes patients are more prone to developing cholelithiasis remains unclear.

In recent years, increasing evidences implicated the role of gut microbiota in the development of multiple chronic disease ${ }^{[10]}$, including non-alcoholic fatty liver disease (NAFLD $^{[11]}$, obesity ${ }^{[12]}$, colorectal cancer ${ }^{[13]}$, diabetes ${ }^{[14]}$ and gallstone disease ${ }^{[15]}$. Thus, gut microbiota homeostasis is crucial to human health. Qin et al.'s study ${ }^{[14]}$ found that opportunistic pathogens such as Escherichia coli, Bacteroides caccae and Clostridium hathewayi were more abundant in type 2 diabetes patients, however butyrate-producing bacteria such as Faecalibacterium prausnitzii and Roseburia intestinalis decreased. Wu et al.'s study ${ }^{[15]}$ showed that the phylum Proteobacteria was significantly increased and the genera Roseburia, Lachnospira and Faecalibacterium were significantly decreased in gallstones patients. Maurer $\mathrm{KJ}^{[16]}$ found that infection with certain strains of Helicobacter promotes gallstone formation in gallstone-susceptible C57L/J mice. The underlying mechanism of gut microbiota in the development of type 2 diabetes and cholelithiasis was not entirely clear. To date, literature has pointed that gut microbiota dysbiosis could lead to the decrease of secondary bile acids, then reduce the activation of bile acid receptors, and finally result in glucose metabolism disorder and type 2 diabetes occurrence ${ }^{[17]}$. Moreover, the research also indicated that gut microbiota could regulate enterohepatic recycling of bile acids, then disrupt the metabolism of cholesterol, and subsequently contribute to the formation of cholesterol gallstones ${ }^{[6,18,19]}$.

Diabetes patients are at a higher risk of developing cholelithiasis ${ }^{[7,8]}$. However, the underlying role of gut microbiota in developing from diabetes to cholelithiasis is still unclear. To this end, 16S rRNA gene sequencing was carried out to analyze the microbial composition of gut from healthy subjects, type 2 diabetes patients, 
cholelithiasis patients and type 2 diabetes complicated with cholelithiasis patients. Clarifying the gut microbial characteristics of patients with type 2 diabetes complicated with cholelithiasis will help improve our understanding of the potential mechanism that diabetes patients are more prone to developing cholelithiasis from the perspective of gut microbiota. This study provides insights into the role of gut microbiota in the progression from diabetes to cholelithiasis.

\section{RESULTS}

\section{Characteristics of clinical samples and data}

In this study, feces samples were collected from 33 healthy subjects (D1 group), 53 type 2 diabetes patients (D2 group), 31 cholelithiasis patients (D3 group) and 32 type 2 diabetes complicated with cholelithiasis patients (D4 group) (see Table 1 for detailed patient information). In total, 149 feces samples were obtained. All cholelithiasis patients were classified as cholesterol gallstones according to the definition proposed by Marschall et al. ${ }^{[20]}$, i.e. a cholesterol content of 50-90\%. When comparing the typical metabolites levels, we found that type 2 diabetes complicated with cholelithiasis patients (D4 group) have rather similar blood glucose levels with type 2 diabetes patients (D2 group) (Figure 1A), but they have higher triglyceride (Figure 1A) and total bile acids levels (Figure 1B) compared with mere cholelithiasis patients (D3 group).

By 16S rRNA gene sequencing, the microbial composition of gut was analyzed. An average of 83,615 raw reads was obtained for per sample. After quality filtering, a mean of 79,042 high-quality reads were retrieved. The sequencing data is enough to reflect species diversity (Supplementary Figure 1). Sequences were clustered into 3030 operational taxonomic units (OTUs ) based on 97\% sequence similarity, with 2232 OTUs in healthy subjects (D1 group), 2246 OTUs in type 2 diabetes patients (D2 group), 1971 OTUs in cholelithiasis patients (D3 group) and 2009 OTUs in type 2 diabetes complicated with cholelithiasis patients (D4 group). The OTUs differences among four groups were statistically significant $(P<0.05$, Kruskal-Wallis test) (Table 1), of which differences between D4 and D1 groups, D4 and D3 groups were statistically significant (D4 vs D1: $P=0.008$, D4 vs D3: $P=0.0051$, Mann-Whitney test, data not shown), and OUT richness was least in D4 group. OTUs were annotated at different classification levels using the Silva132 database. At the phylum level, three phyla predominated: Firmicutes, Bacteroidetes and Proteobacteria. 
111 Microbial diversity significantly decreased in type 2 diabetes complicated with cholelithiasis patients

113 The intra-community alpha diversity, which represents microbial diversity, was

114 analyzed. The observed species, which represent microbial richness, were significantly

115 decreased in type 2 diabetes complicated with cholelithiasis patients (D4 group)

116 compared with cholelithiasis patients (D3 group) ( $P=0.0031$, Wilcoxon rank-sum test)

117 and healthy subjects (D1 group) ( $P=0.0179$, Wilcoxon rank-sum test) (Figure 2A). The

118 shannon index between D4 and D3 groups ( $P=0.0224$, Wilcoxon rank-sum test), D4

119 and D2 groups ( $P=0.0159$, Wilcoxon rank-sum test), D4 and D1 groups $(P=0.0091$,

120 Wilcoxon rank-sum test) were also statistically different (Figure 2B). Patients with type

1212 diabetes complicated with cholelithiasis (D4 group) presented the lowest shannon

122 index. The results suggested that gut microbiota dysbiosis was more intricate in patients

123 with type 2 diabetes complicated with cholelithiasis. For other alpha diversity indices,

124 such as simpson, chao 1, good's coverage and PD_whole tree, consistent results were

125 obtained (data not shown).

126 The beta diversity was also analyzed to discover whether gut microbial community 127 differences were significantly existed between groups. We found that unweighted

128 unifrac based-beta diversity between D4 and D3 groups, D4 and D1 groups were 129 significantly different $(P<0.01$, Wilcoxon rank-sum test), but there was no statistical 130 difference between D4 and D2 groups ( $P=0.3858$, Wilcoxon rank-sum test) (Figure 2C).

131 Moreover, principal co-ordinates analysis (PCoA) using unweighted unifrac distance 132 algorithm showed that the microbial community structureof type 2 diabetes 133 complicated with cholelithiasis patients (D4 group) was similar with type 2 diabetes 134 patients (D2 group) $(P=0.051$, AMOVA), whereas was significant different from 135 cholelithiasis patients (D3 group) ( $P=0.001$, AMOVA) (Figure 2D). This finding 136 suggested that type 2 diabetes complicated with cholelithiasis might share common gut 137 microbial characteristics with type 2 diabetes.

138 Type 2 diabetes, cholelithiasis and type 2 diabetes complicated with cholelithiasis 139 remodeled the gut microbial communities

140 At the phylum level, the relative abundance of Firmicutes, Bacteroidetes and 141 Proteobacteria was more than $96 \%$ in all groups. Firmicutes was most common in 142 healthy subjects (D1 group) (approximately 57.75\%), but decreased to 48.23\%, 44.64\% 143 and $44.60 \%$ in type 2 diabetes patients (D2 group), cholelithiasis patients (D3 group) 
144 and type 2 diabetes complicated with cholelithiasis patients (D4 group), respectively 145 (Figure 3A). In contrast, Bacteroidetes increased from $32.89 \%$ in healthy subjects (D1 146 group) to $39.24 \%, 43.87 \%$ and $36.44 \%$, Proteobacteria increased from $6.91 \%$ in 147 healthy subjects (D1 group) to $9.12 \%, 8.53 \%$ and $15.95 \%$, in type 2 diabetes patients 148 (D2 group), cholelithiasis patients (D3 group) and type 2 diabetes complicated with 149 cholelithiasis patients (D4 group), respectively. Verrucomicrobia slightly increased in 150 cholelithiasis patients (D3 group) and type 2 diabetes complicated with cholelithiasis 151 patients (D4 group) (Figure 3A). The gut microbial composition of all samples at the 152 phylum level were shown in Supplementary Figure 2.

153 Among the top 10 genera, Bacteroides predominated, which increased from $26.46 \%$ 154 in healthy subjects (D1 group) to $29.46 \%, 37.00 \%$ and $26.55 \%$ in type 2 diabetes 155 patients (D2 group), cholelithiasis patients (D3 group) and type 2 diabetes complicated 156 with cholelithiasis patients (D4 group), respectively. The genera Faecalibacterium and 157 Megamonas were most common in healthy subjects (D1 group), Faecalibacterium 158 decreased from $11.30 \%$ in healthy subjects (D1 group) to $5.89 \%, 7.65 \%$ and $5.57 \%$, 159 Megamonas decreased from $4.44 \%$ in healthy subjects (D1 group) to $0.53 \%, 0.44 \%$ and $1600.87 \%$, in type 2 diabetes patients (D2 group), cholelithiasis patients (D3 group) and 161 type 2 diabetes complicated with cholelithiasis patients (D4 group), respectively 162 (Figure 3B). The relative abundance of genus unidentified Enterobacteriaceae 163 increased from $1.47 \%$ in healthy subjects (D1 group) to $3.38 \%, 4.23 \%$ and $5.40 \%$ in 164 type 2 diabetes patients (D2 group), cholelithiasis patients (D3 group) and type 2 165 diabetes complicated with cholelithiasis patients (D4 group), respectively (Figure 3B).

166 The gut microbial composition of all samples at the genus level were shown in 167 Supplementary Figure 3.

168 Similarity and association analysis of gut microbial composition

169 To further investigate the similarities and associations of gut microbial 170 communities, unweighted pair group method with arithmetic mean (UPGMA, 171 weighted unifrac) clustering analysis at the phylum level was performed. The resulted 172 cluster tree showed that four groups were divided into three different clusters, type 2 173 diabetes complicated with cholelithiasis patients (D4 group) was in cluster A, type 2 174 diabetes patients (D2 group) and cholelithiasis patients (D3 group) were in cluster B, 175 and healthy subjects (D1 group) was in cluster C (Figure 3C). The results suggested that 176 the gut microbial composition of mere type 2 diabetes and mere cholelithiasis was 
similar at the phylum level, whereas some alterations were generated in type 2 diabetes complicated with cholelithiasis.

179 Furthermore, Venn diagram analysis showed that 68 OTUs were common to type 2 180 diabetes (D2 group) and cholelithiasis (D3 group), 94 OTUs were common to type 2 181 diabetes (D2 group) and type 2 diabetes complicated with cholelithiasis (D4 group), 52 182 OTUs were common to cholelithiasis (D3 group) and type 2 diabetes complicated with 183 cholelithiasis (D4 group), and 70 OTUs were shared by D2, D3 and D4 groups (Figure 184 3D). Among the 70 OTUs, the phyla Firmicutes, Bacteroidetes, Proteobacteria and 185 Actinobacteria, and the genera Sutterella, Bacteroides, Dialister and Parasutterella, 186 were significantly different between D2, D3, D4, and D1 groups (data not shown).

\section{Differences in gut microbial components}

188 The linear discriminant analysis (LDA) effect size (LEfSe) analysis according to 189 LDA scores was performed by using non-parametric Kruskal-Wallis rank-sum test to 190 detect species with significant differences between groups. The results showed that as 191 compared with healthy subjects (D1 group), the phylum Proteobacteria, class 192 Gammaproteobacteria and order Lactobacillales were more abundant in type 2 193 diabetes patients (D2 group), whereas the genus Faecalibacterium was lower (Figure 194 4A and 4B). In cholelithiasis patients (D3 group), the relative abundances of 195 Bacteroidetes taxa groups including class Bacteroidia, order Bacteroidales, family 196 Bacteroidaceae and genus Bacteroides were significantly increased, and the phylum 197 Firmicutes and genus Faecalibacterium were significantly decreased (Figure 4C and 198 4D). In patients with type 2 diabetes complicated with cholelithiasis (D4 group), both 199 Proteobacteria taxa groups including class Gammaproteobacteria, order 200 Enterobacteriales, family Enterobacteriaceae and genus unidentified 201 Enterobacteriaceae, and class Bacilli taxa groups including order Lactobacillales, 202 family Lactobacillaceae and genus Lactobacillus, were significantly enriched. On the 203 contrary, Firmicutes taxa groups including class Clostridia, order Clostridiales, family 204 Ruminococcaceae, genera Faecalibacterium, Megamonas and Subdoligranulum were 205 significantly decreased (Figure 4E and 4F).

As compared with type 2 diabetes patients (D2 group), the Proteobacteria taxa groups which includes class Gammaproteobacteria, order Enterobacteriales and

208 family Enterobacteriaceae were significantly increased in type 2 diabetes complicated 209 with cholelithiasis patients (D4 group) (Figure 5A and 5B). However, there exists more 210 species with significant differences between cholelithiasis patients (D3 group) and type 
2112 diabetes complicated with cholelithiasis patients (D4 group) (Figure 5C and 5D).

212 From these results, we found that the tendency of gut microbiota alterations in type 2

213 diabetes complicated with cholelithiasis patients was more similar with mere type 2

214 diabetes patients, rather than cholelithiasis patients.

\section{DISCUSSION}

216 Several studies have shown that gut microbiota is partially associated with the 217 pathogenesis of type 2 diabetes $^{[14]}$ and cholelithiasis ${ }^{[15]}$. Epidemiological studies 218 indicated that diabetes patients have an increased risk of gallstones formation ${ }^{[7,8]}$. 219 However, no association study has been carried out to clarify the underlying role of gut 220 microbiota in developing from type 2 diabetes to cholelithiasis. This study 221 demonstrated the intricate gut microbiota dysbiosis present with type 2 diabetes 222 complicated with cholelithiasis and discovered an underlying association between type 2232 diabetes and type 2 diabetes complicated with cholelithiasis, that is, gut microbiota 224 has the potential to promote type 2 diabetes progressing to type 2 diabetes complicated 225 with cholelithiasis. This is the first study to clarify the microbial composition of gut 226 with type 2 diabetes complicated with cholelithiasis. The results of this study suggested 227 that the gut microbiota alterations of type 2 diabetes complicated with cholelithiasis 228 patients was similar but more intricate compared with mere type 2 diabetes patients, 229 which were supported with methods such as PCoA analysis, UPGMA analysis and 230 LEfSe analysis, as will be discussed in the following.

231 This study discovered similar but more intricate changes of gut microbial 232 composition in type 2 diabetes complicated with cholelithiasis patients compared with 233 mere type 2 diabetes patients. Unweighted unifrac-based PCoA analysis identified a 234 high degree of microbial community structure similarity between type 2 diabetes 235 complicated with cholelithiasis patients and type 2 diabetes patients (Figure 2D). 236 Meanwhile, 94 OTUs were shared by type 2 diabetes complicated with cholelithiasis 237 patients and type 2 diabetes patients by Venn diagram (Figure 4B). However, weighted 238 unifrac-based UPGMA analysis at the phylum level showed that type 2 diabetes 239 complicated with cholelithiasis patients and type 2 diabetes patients were grouped into 
240 different clusters (Figure 4A), suggesting there exists unique gut microbial components, 241 which might complicate the gut microbiota dysbiosis. Interestingly, serum triglyceride 242 and total bile acids levels were significantly increased compared with mere 243 cholelithiasis patients (Figure 1). The abnormal metabolism condition indirectly 244 reflects the intricate gut microbiota dysbiosis within type 2 diabetes complicated with 245 cholelithiasis patients.

246 Within the gut of cholelithiasis patients, the Bacteroidetes taxa groups which 247 includes various pathogens such as Bacteroidia, Bacteroidales, Bacteroidaceae and 248 Bacteroides were significantly increased, the phylum Firmicutes and genus 249 Faecalibacterium were notably decreased (Figure 5C and D). However, within the gut 250 of type 2 diabetes complicated with cholelithiasis patients, Proteobacteriataxa groups 251 including Gammaproteobacteria, Enterobacteriales, Enterobacteriaceae and 252 unidentified Enterobacteriaceae were significantly enriched, the phylum Firmicutes 253 and genera Faecalibacterium, Megamonas and Subdoligranulum were significantly 254 decreased (Figure 5E and F), which were quite different from mere cholelithiasis 255 patients but partial similar with mere type 2 diabetes patients (Figure 5A and B). 256 Furthermore, we found that there exists an increase of the phylum Proteobacteria taxa 257 groups which includes Gammaproteobacteria, Enterobacteriales and 258 Enterobacteriaceae in type 2 diabetes complicated with cholelithiasis patients 259 compared with mere type 2 diabetes patients (Figure 6A and B), which mainly 260 containing opportunistic pathogenic bacteria of human beings. The results suggested 261 that type 2 diabetes complicated with cholelithiasis patients had similar but more 262 intricate gut microbiota dysbiosis compared with mere type 2 diabetes patients. These 263 bacterial species with significant differences might have a potential association with 264 cholelithiasis occurrence in type 2 diabetes patients.

265 Gut microbiota can regulate bile acids metabolism ${ }^{[21]}$. The abnormal metabolism of 266 bile acids is the main pathogenesis of cholesterol gallstones ${ }^{[6]}$. Several studies have 267 indicated that gut microbiota can suppress bile acids synthesis through activating the 
nuclear receptor Farnesol $\mathrm{X}$ receptor $(\mathrm{FXR})^{[22,23]}$. The phyla Actinobacteria,

269 Proteobacteria, Firmicutes and Bacteroidetes can produce hydroxysteroid

270 dehydrogenases (HSDHs), which can catalyze bile acids into oxo- (or keto-) bile 271 acids $^{[24-26]}$. Some bacteria such as Lactobacilli, Bifidobacteria and Bacteroides can 272 secrete bile salt hydrolase (BSH) and then de-conjugate bile acids, thus regulate the 273 microbial metabolism of bile acids ${ }^{[27-29]}$. In this study, the relative abundance of 274 Lactobacillus, Bifidobacterium and Bacteroides were increased in the gut of type 2 275 diabetes patients, suggesting that the changes of bacteria with $\mathrm{BSH}$ activity in type 2 276 diabetes patients might lead to the abnormal metabolism of bile acids and subsequently 277 contribute to the pathogenesis of cholelithiasis, which explained that diabetes patients 278 are at an increased risk of developing cholelithiasis from the perspective of gut 279 microbiota. However, further research is still needed to investigate the effect of these 280 bacteria on gallstone formation.

\section{CONCLUSION}

282 Gut microbial characteristics and abnormal metabolism condition in type 2 diabetes 283 complicated with cholelithiasis patients was first discovered in this study, that is, the 284 gut microbial composition was partial similar with type 2 diabetes patients, whereas 285 present with more intricate gut microbiota dysbiosis. The results of this study suggested 286 that there exists certain bacterial species in type 2 diabetes patients, which are 287 associated with the occurrence of cholelithiasis. Thus, maintaining the gut microbiota 288 homeostasis of type 2 diabetes patients might help reduce the risk of subsequently 289 developing cholelithiasis.

\section{METHODS}

\section{$291 \quad$ Patients and feces collection}

292 From September 2017 to September 2018, 53 patients with mere type 2 diabetes 293 from the department of endocrinology, 31 patients with mere cholelithiasis and 32 294 patients with type 2 diabetes complicated with cholelithiasis from the department of 295 general surgery, and 33 healthy subjects from the department of physical examination 
were enrolled, with average age of $58.5 \pm 9.6$ and a male ratio of $45.6 \%$. None of patients

297 indicated that they had any other infectious or underlying diseases. None of the healthy 298 subjects indicated that they had suffered type 2 diabetes, cholelithiasis, or any other 299 infectious and metabolic diseases. All participants have not been treated with antibiotics 300 in the last three months. The metabolic indexes were derived from retrospective medical 301 records. Feces samples from all patients and healthy subjects were collected and placed 302 in sterile Eppendorf tube, glycerin was added to final concentration of $20 \%$. All feces 303 samples were stored at $-80^{\circ} \mathrm{C}$.

\section{DNA preparation, library construction, and sequencing}

305 Genomic DNA was extracted from feces samples using the QIAamp DNA Stool 306 Mini Kit (Qiagen) and then agarose gel electrophoresis was performed to detect the 307 purity and concentration of extracted DNA. As template, the qualified genomic DNA 308 was prepared with concentration of $1 \mathrm{ng} / \mu \mathrm{l}$ to amplify gene sequence in V4 region of 309 bacteria 16S ribosomal RNA. The forward primer (515F) was 310 5'-GTGCCAGCMGCCGCGGTAA-3'. The reverse primer (806R) was 311 5'-GGACTACHVGGGTWTCTAAT-3'. Then the PCR products were mixed with equal 312 amounts and purified by GeneJETTM PCR Purification Kit (Thermo). DNA libraries 313 were prepared with Ion Plus Fragment Library Kit (Thermo) and then sequenced on 314 Thermoscher's Ion S5TM XL platform. After shearing the low-quality reads by using 315 Cutadapt (V1.9.1), the sample data was separated from the obtained reads according to 316 barcode. The barcode and primer sequences were truncated to obtain the raw data (raw 317 reads). Then, the chimeric sequence was detected and removed by comparing raw reads 318 sequence with species annotation database according to UCHIME Algorithm. Finally, 319 the final effective data was obtained (clean reads).

\section{Processing of sequencing data and bioinformatics analysis}

321 All clean reads data were clustered as OTUs according to 97\% identity. Species 322 annotation of these OTUs were performed using mothur method (with a threshold of 323 0.8 1). Classification information at each level were obtained: from kingdom to species. 
324 Finally, the data of each sample were homogenized. QIIME (version 1.9.1) was used to

325 calculate community diversity index and unifrac distance (alpha diversity and beta

326 diversity analysis). The rarefaction curve, rank abundance curve, species accumulation

327 curve, microbial abundance histogram, Venn diagram and UPGMA group clustering tree

328 were drawn using $\mathrm{R}$ software (version 2.15.3). LEfSe analysis was performed using

329 LEfSe software with a default setting of LDA Score $=4$.

$330 \quad$ Statistical analyses

331 SPSS 16.0 (SPSS, Inc., Chicago, IL, USA) and R software (version 2.15.3) were 332 used for statistical analysis. General characteristics are expressed as mean \pm standard 333 deviation (SD), mean \pm standard error of mean (SEM) or percentage. Kruskal-Wallis 334 non-parametric test was used for multiple group comparison. Student's t-test and 335 Wilcoxon rank-sum testwere used for two group comparison. $P<0.05$ was considered 336 statistically significant.

\section{Ethics}

339 The study was approved by the Ethics Committee of Peking University Shougang 340 Hospital (SGYYZ201701). All study participants provided informed written consent 341 prior to study enrollment.

\section{Funding}

343 This research was funded by the Beijing Natural Science Foundation [grant number 344 7192240], National Natural Science Foundation of China [grant number 81900386], 345 and Peking University Shougang Hospital [grant number SGYYZ201701, 346 SGYYQ201903]

\section{Author contributions}

$348 \mathrm{Gu} \mathrm{J}$ and Hu SK designed and coordinated the study; Chen JJ, Yan LL and Ma XF 349 performed the experiments; Chen JJ and Yan LL analyzed the data and wrote the 350 manuscript; Yuan P, Zhao F, Han ZH, Liu JS, Wang WB, Zhou DH, Zhao HY, Feng N 351 and Huang DD contributed reagents and materials. 
353 The authors declare that they have no conflicts of interest.

354 Supplementary Material:

355 Figure S1 Quality analysis of sequencing data.

356 Figure S2 Relative abundance of each sample at the phylum level.

357 Figure S3 Relative abundance of each sample at the genus level.

\section{REFERENCES}

3601 World Health Organization. http://apps. who.int/ghodata/?vid=2469.. 2008

3612 Portincasa P, Moschetta A, Palasciano G. Cholesterol gallstone disease. Lancet 362 2006; 368: 230-239 [PMID: 16844493 DOI: 10.1016/S0140-6736(06)69044-2]

3633 Sandler RS, Everhart JE, Donowitz M, Adams E, Cronin K, Goodman C, 364 Gemmen E et al. The burden of selected digestive diseases in the United States. 365 Gastroenterology 2002; 122: 1500-1511 [PMID: 11984534 DOI: $366 \quad 10.1053 /$ gast.2002.32978]

3674 Yang W, Lu J, Weng J, Jia W, Ji L, Xiao J, Shan Z, et al. China National Diabetes 368 and Metabolic Disorders Study Group. Prevalence of diabetes among men and 369 women in China. N Engl J Med 2010; 362: 1090-1101 [PMID: 20335585 DOI: 370 10.1056/NEJMoa0908292]

3715 Mendez-Sanchez N, Chavez-Tapia NC, Motola-Kuba D, Sanchez-Lara K, 372 Ponciano-Rodriguez G, Baptista H, Ramos MH et al. Metabolic syndrome as a 373 risk factor for gallstone disease. World J Gastroenterol 2005; 11: 1653-1657 374 [PMID: 15786544 DOI:10.3748/wjg.v11.i11.1653 ]

3756 Wang DQ, Cohen DE, Carey MC. Biliary lipids and cholesterol gallstone disease. 376 J Lipid Res 2009; 50 Suppl: S406-411 [PMID: 19017613 DOI: 377 10.1194/jlr.R800075-JLR200]

3787 Haffner SM, Diehl AK, Mitchell BD, Stern MP, Hazuda HP. Increased prevalence 379 of clinical gallbladder disease in subjects with non-insulin-dependent diabetes 
mellitus. Am J Epidemiol 1990; 132: 327-335 [PMID: 2196792 DOI:

$381 \quad$ 10.1093/oxfordjournals.aje.a115662]

3828 Ruhl CE, Everhart JE. Association of diabetes, serum insulin, and C-peptide with 383 gallbladder disease. Hepatology 2000; 31: 299-303 [PMID: 10655249 DOI: $384 \quad 10.1002 /$ hep.510310206]

3859 Biddinger SB, Haas JT, Yu BB, Bezy O, Jing E, Zhang W, Unterman TG et al. 386 Hepatic insulin resistance directly promotes formation of cholesterol gallstones. 387 Nat Med 2008; 14: 778-782 [PMID: 18587407 DOI: 10.1038/nm1785]

38810 Gilbert JA, Blaser MJ, Caporaso JG, Jansson JK, Lynch SV, Knight R. Current 389 understanding of the human microbiome. Nat Med 2018; 24: 392-400 [PMID: $390 \quad 29634682$ DOI: $10.1038 / \mathrm{nm} .4517]$

39111 Henao-Mejia J, Elinav E, Jin C, Hao L, Mehal WZ, Strowig T, Thaiss CA et al. 392 Inflammasome-mediated dysbiosis regulates progression of NAFLD and obesity. 393 Nature 2012; 482: 179-185 [PMID: 22297845 DOI: 10.1038/nature10809]

39412 Bajzer M, Seeley RJ. Physiology: obesity and gut flora. Nature 2006; 444: 395 1009-1010 [PMID: 17183300 DOI: 10.1038/4441009a]

39613 Wong SH, Zhao L, Zhang X, Nakatsu G, Han J, Xu W, Xiao X et al. Gavage of 397 Fecal Samples From Patients With Colorectal Cancer Promotes Intestinal 398 Carcinogenesis in Germ-Free and Conventional Mice. Gastroenterology 2017; 399 153: 1621-1633 e1626 [PMID: 28823860 DOI: 10.1053/j.gastro.2017.08.022]

40014 Qin J, Li Y, Cai Z, Li S, Zhu J, Zhang F, Liang S et al. A metagenome-wide $401 \quad$ association study of gut microbiota in type 2 diabetes. Nature 2012; 490: 55-60 402 [PMID: 23023125 DOI: 10.1038/nature11450]

40315 Wu T, Zhang Z, Liu B, Hou D, Liang Y, Zhang J, Shi P. Gut microbiota dysbiosis 404 and bacterial community assembly associated with cholesterol gallstones in 405 large-scale study. BMC Genomics 2013; 14: 669 [PMID: 24083370 DOI: $406 \quad 10.1186 / 1471-2164-14-669]$ 
16 Maurer KJ, Ihrig MM, Rogers AB, Ng V, Bouchard G, Leonard MR, Carey MC et 408 al. Identification of cholelithogenic enterohepatic helicobacter species and their 409 role in murine cholesterol gallstone formation. Gastroenterology 2005; 128: 410 1023-1033 [PMID: 15825083 DOI: 10.1053/j.gastro.2005.01.008]

41117 Ma Q, Li Y, Li P, Wang M, Wang J, Tang Z, Wang T et al. Research progress in 412 the relationship between type 2 diabetes mellitus and intestinal flora. Biomed 413 Pharmacother 2019; 117: 109138 [PMID: 31247468 DOI: $414 \quad$ 10.1016/j.biopha.2019.109138]

41518 Sayin SI, Wahlstrom A, Felin J, Jantti S, Marschall HU, Bamberg K, Angelin B, et 416 al. Gut microbiota regulates bile acid metabolism by reducing the levels of 417 tauro-beta-muricholic acid, a naturally occurring FXR antagonist. Cell Metab 418 2013; 17: 225-235 [PMID: 23395169 DOI: 10.1016/j.cmet.2013.01.003]

41919 Keren N, Konikoff FM, Paitan Y, Gabay G, Reshef L, Naftali T, Gophna U. 420 Interactions between the intestinal microbiota and bile acids in gallstones patients. 421 Environ Microbiol Rep 2015; 7: 874-880 [PMID: 26149537 DOI: $422 \quad 10.1111 / 1758-2229.12319]$

42320 Marschall HU, Einarsson C. Gallstone disease. J Intern Med 2007; 261: 529-542 424 [PMID: 17547709 DOI: 10.1111/j.1365-2796.2007.01783.x]

42521 Jia W, Xie G, Jia W. Bile acid-microbiota crosstalk in gastrointestinal 426 inflammation and carcinogenesis. Nat Rev Gastroenterol Hepatol 2018; 15: 427 111-128 [PMID: 29018272 DOI: 10.1038/nrgastro.2017.119]

42822 Gerard P. Metabolism of cholesterol and bile acids by the gut microbiota. 429 Pathogens 2013; 3: 14-24 [PMID: 25437605 DOI: 10.3390/pathogens3010014] 43023 Lefebvre P, Cariou B, Lien F, Kuipers F, Staels B. Role of bile acids and bile acid 431 receptors in metabolic regulation. Physiol Rev 2009; 89: 147-191 [PMID: 432 19126757 DOI: 10.1152/physrev.00010.2008] 
24 Kisiela M, Skarka A, Ebert B, Maser E. Hydroxysteroid dehydrogenases (HSDs)

434 in bacteria: a bioinformatic perspective. J Steroid Biochem Mol Biol 2012; 129:

435 31-46 [PMID: 21884790 DOI: 10.1016/j.jsbmb.2011.08.002]

43625 Fukiya S, Arata M, Kawashima H, Yoshida D, Kaneko M, Minamida K, 437 Watanabe $\mathrm{J}$ et al. Conversion of cholic acid and chenodeoxycholic acid into their 438 7-oxo derivatives by Bacteroides intestinalis AM-1 isolated from human feces. 439 FEMS Microbiol Lett 2009; 293: 263-270 [PMID: 19243441 DOI: $440 \quad$ 10.1111/j.1574-6968.2009.01531.x]

44126 Hirano S, Masuda N. Epimerization of the 7-hydroxy group of bile acids by the 442 combination of two kinds of microorganisms with 7 alpha- and 7 443 beta-hydroxysteroid dehydrogenase activity, respectively. J Lipid Res 1981; 22: 444 1060-1068 [PMID: 6946176]

44527 Gilliland SE, Speck ML. Deconjugation of bile acids by intestinal lactobacilli. 446 Appl Environ Microbiol 1977; 33: 15-18 [PMID: 13710]

44728 Jones BV, Begley M, Hill C, Gahan CG, Marchesi JR. Functional and 448 comparative metagenomic analysis of bile salt hydrolase activity in the human gut 449 microbiome. Proc Natl Acad Sci U S A 2008; 105: 13580-13585 [PMID: 450 18757757 DOI: 10.1073/pnas.0804437105]

45129 Ridlon JM, Kang DJ, Hylemon PB. Bile salt biotransformations by human 452 intestinal bacteria. J Lipid Res 2006; 47: 241-259 [PMID: 16299351 DOI: 453 10.1194/jlr.R500013-JLR200] 


\section{Figure Legends}

Figure 1: Serum metabolites levels. A: Blood glucose, total cholesterol and triglyceride levels in D2, D3 and D4 groups; B: Total bile acids levels in D3 and D4 groups. The levels were expressed as mean \pm SEM. (D2: Type 2 diabetes; D3: Cholelithiasis; D4: type 2 diabetes complicated with cholelithiasis. ${ }^{\mathrm{a}} P<0.05,{ }^{\mathrm{b}} P<0.01$, ' $P<0.001$, Unpaired Student's t-test.)

Figure 2: Gut microbial diversity analysis. A: Observed species, the scatter distribution of total number of species, namely the richness; B: Shannon index, namely species diversity and evenness; C: Unweighted unifrac based-beta diversity analysis, assessing gut microbial community differences; D: PCoA, showing the microbial community structure similarity. (D1: Healthy subjects; D2: Type 2 diabetes; D3: Cholelithiasis; D4: type 2 diabetes complicated with cholelithiasis. ${ }^{\mathrm{a}} P<0.05,{ }^{\mathrm{b}} P<0.01$, ${ }^{c} P<0.001$; A, B, C: Wilcoxon rank-sum test; D: AMOVA)

Figure 3: The composition of gut microbiota in 4 groups. A: Showing the top 10 phyla; B: Showing the top 10 genera. C: Weighted unifrac distance based-UPGMA cluster tree analysis at the phylum level, left: UPGMA clustering tree structure, right: relative abundance distribution of species; D: Unique and shared OTUs of gut microbiota among four groups are represented by Venn diagram. These numbers show the number of OTUs in different groups. There is a total of 3006 OTUs. (D1: Healthy subjects; D2: Type 2 diabetes; D3: Cholelithiasis; D4: type 2 diabetes complicated with cholelithiasis.)

\section{Figure 4: Different structures of gut microbiota in patients and healthy subjects} by LEfSe analysis. A,C,E: Specific species of gut microbiota between two groups by LEfSe analysis, the histogram shows the LDA scores, the lateral text shows the different species between two groups; B,D,F: LEfSe cladogram, showing the different species from phylum level (outer circle) to species level (inner circle), the red and green cladogram represent different groups, the abundance is proportional to the diameter of circle. A,B: D2 vs D1 groups; C,D: D3 vs D1 groups; E,F: D4 vs D1 groups. LDA SCORE=4. (D1: Healthy subjects; D2: Type 2 diabetes; D3: Cholelithiasis; D4: type 2 diabetes complicated with cholelithiasis.)

\section{Figure 5: Different structures of gut microbiota among disease groups by LEfSe} analysis. A,C: Specific species of gut microbiota between two groups by LEfSe analysis, the histogram shows the LDA scores, the lateral text shows the different 
488 species between two groups; B,D: LEfSe cladogram, showing the different species 489 from phylum level (outer circle) to species level (inner circle), the red and green 490 cladogram represent different groups, the abundance is proportional to the diameter of 491 circle. A,B: D4 vs D2 groups; C,D: D4 vs D3 groups. LDA SCORE = 4. (D2: Type 2 492 diabetes; D3: Cholelithiasis; D4: type 2 diabetes complicated with cholelithiasis.) 
Table 1 Characteristics of clinical samples and data

\begin{tabular}{|c|c|c|c|c|c|}
\hline & $\begin{array}{l}\text { D1 group } \\
\text { (healthy } \\
\text { subjects, } \\
\text { n=33) }\end{array}$ & $\begin{array}{l}\text { D2 group } \\
\text { (type } 2 \\
\text { diabetes, } \\
\text { n=53) }\end{array}$ & $\begin{array}{l}\text { D3 group } \\
\text { (cholelithiasis, } \\
\text { n=31) }\end{array}$ & $\begin{array}{l}\text { D4 group } \\
\text { (type } 2 \text { diabetes } \\
\text { complicated with } \\
\text { cholelithiasis, } n=32 \text { ) }\end{array}$ & $\begin{array}{l}\text { Total } \\
(n=149)\end{array}$ \\
\hline $\begin{array}{l}\text { Age } \\
(\text { mean } \pm \\
\text { SD) }\end{array}$ & $51.0 \pm 4.5$ & $61.6 \pm 9.7$ & $60.1 \pm 11.0$ & $59.5 \pm 7.8$ & $58.5 \pm 9.6$ \\
\hline $\begin{array}{l}\text { Male } \\
(\text { no. ,\%) }\end{array}$ & $17,51.5 \%$ & $29,54.7 \%$ & $8,25.8 \%$ & $14,43.8 \%$ & $68,45.6 \%$ \\
\hline $\begin{array}{l}\text { Total } \\
\text { OTUs }\end{array}$ & 2232 & 2246 & 1971 & 2009 & 3030 \\
\hline $\begin{array}{l}\text { OTUs } \\
\text { per } \\
\text { samples* }\end{array}$ & $705.0 \pm 122.7$ & $661.1 \pm 114.5$ & $700.5 \pm 94.0$ & $622.9 \pm 117.3$ & $670.8 \pm 116.2$ \\
\hline
\end{tabular}



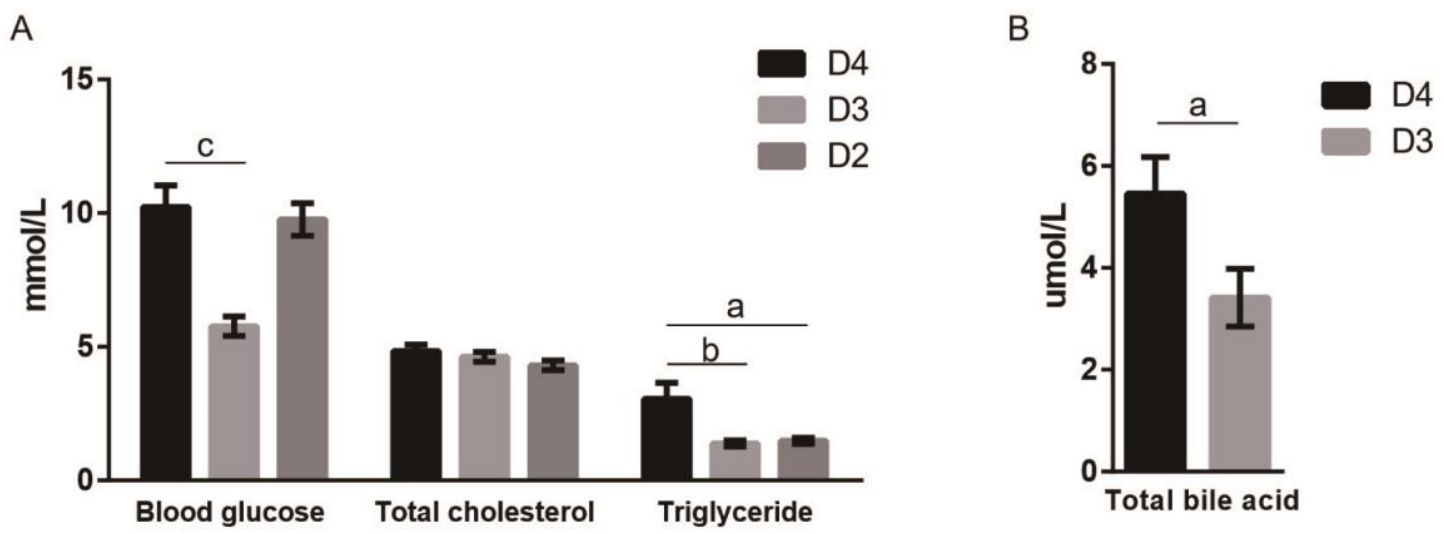

Figure 1: Serum metabolites levels. A: Blood glucose, total cholesterol and triglyceride levels in D2, D3 and D4 groups; B: Total bile acids levels in D3 and D4 groups. The levels were expressed as mean \pm SEM. (D2: Type 2 diabetes; D3: Cholelithiasis; D4: type 2 diabetes complicated with cholelithiasis. ${ }^{\mathrm{a}} P<0.05,{ }^{\mathrm{b}} P<0.01$, ${ }^{\mathbf{c}} P<0.001$, Unpaired Student's t-test.) 


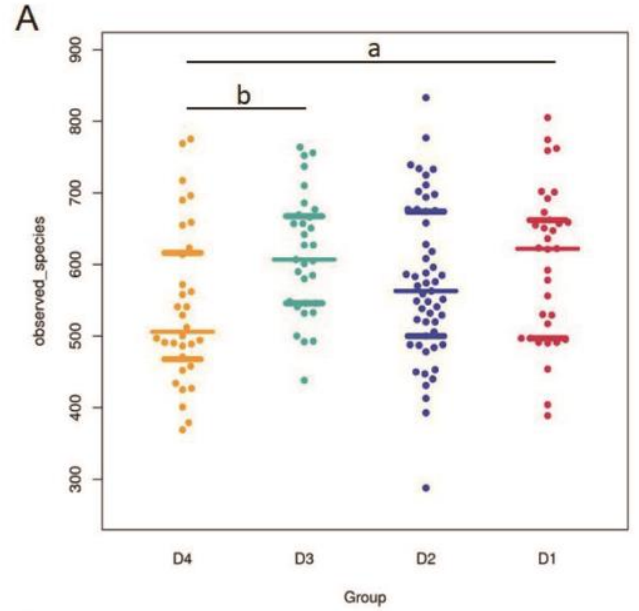

C

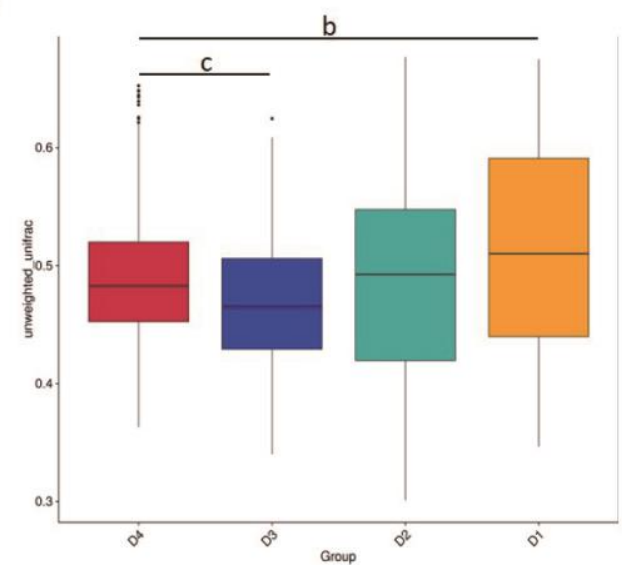

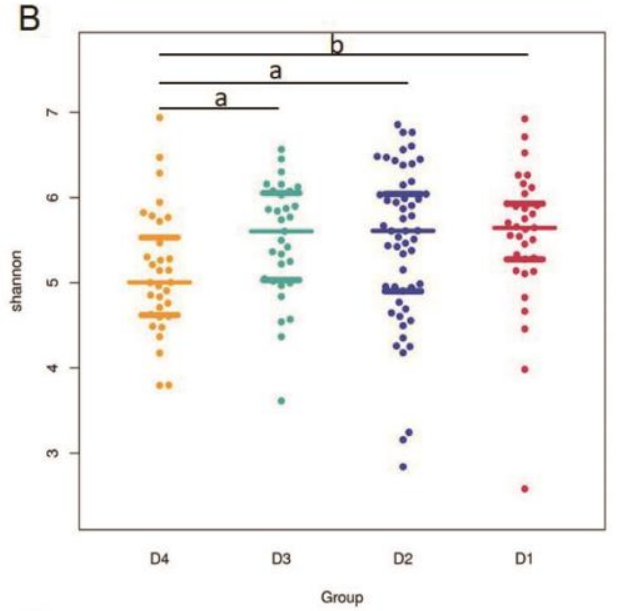

D
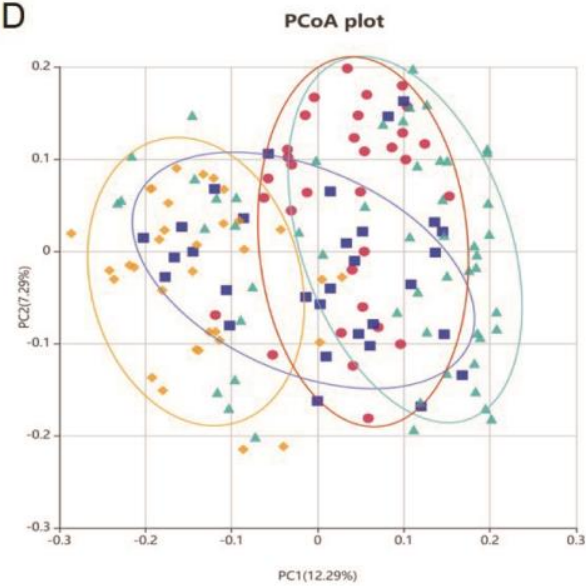

Figure 2: Gut microbial diversity analysis. A: Observed species, the scatter distribution of total number of species, namely the richness; B: Shannon index, namely species diversity and evenness; C: Unweighted unifrac based-beta diversity analysis, assessing gut microbial community differences; D: PCoA, showing the microbial community structure similarity. (D1: Healthy subjects; D2: Type 2 diabetes; D3: Cholelithiasis; D4: type 2 diabetes complicated with cholelithiasis. ${ }^{\mathrm{a}} P<0.05,{ }^{\mathrm{b}} P<0.01$, ' $P<0.001$; A, B, C: Wilcoxon rank-sum test; D: AMOVA) 

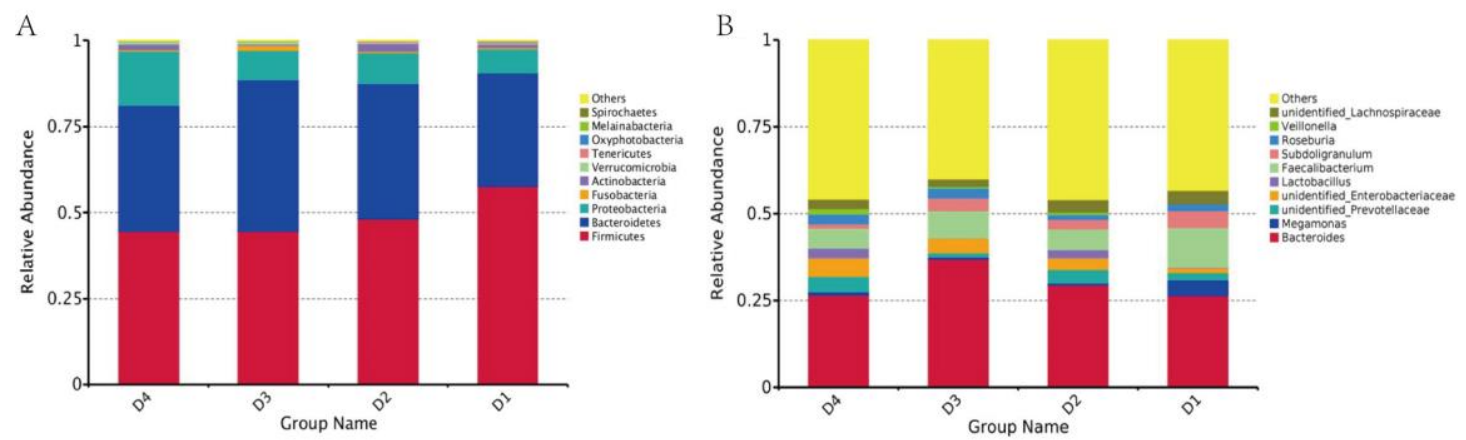

C

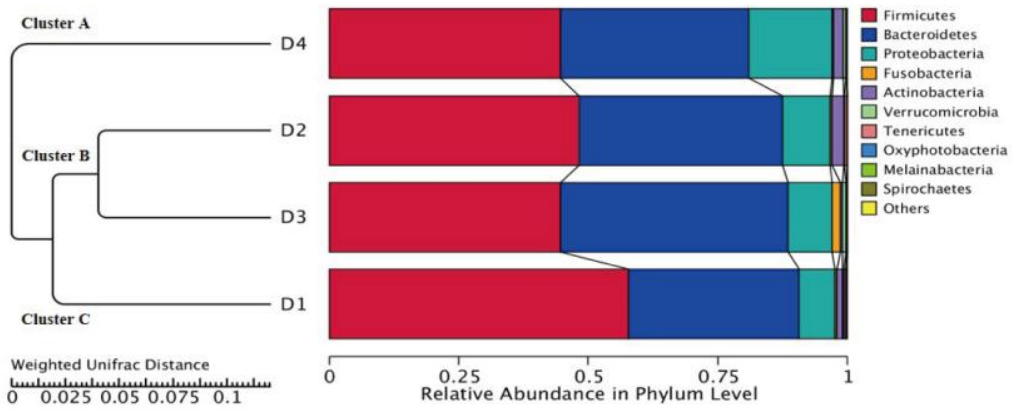

D

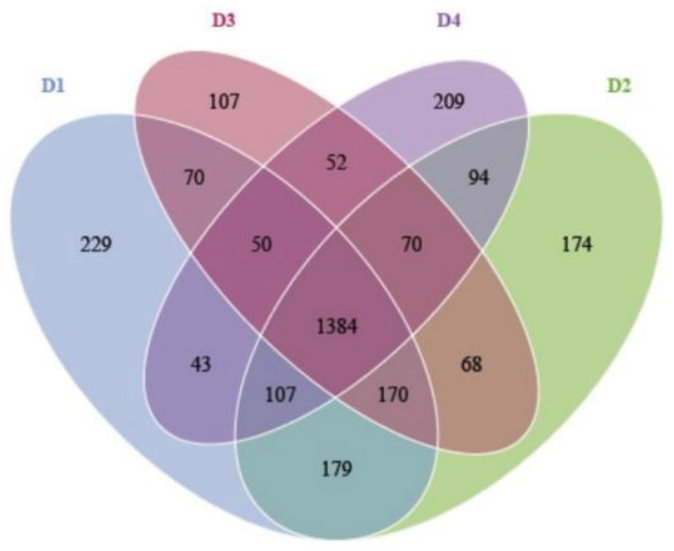

Figure 2: Gut microbial diversity analysis. A: Observed species, the scatter distribution of total number of species, namely the richness; B: Shannon index, namely species diversity and evenness; C: Unweighted unifrac based-beta diversity analysis, assessing gut microbial community differences; D: PCoA, showing the microbial community structure similarity. (D1: Healthy subjects; D2: Type 2 diabetes; D3: Cholelithiasis; D4: type 2 diabetes complicated with cholelithiasis. ${ }^{\mathrm{a}} P<0.05,{ }^{\mathrm{b}} P<0.01$, ' $P<0.001$; A, B, C: Wilcoxon rank-sum test; D: AMOVA) 
A

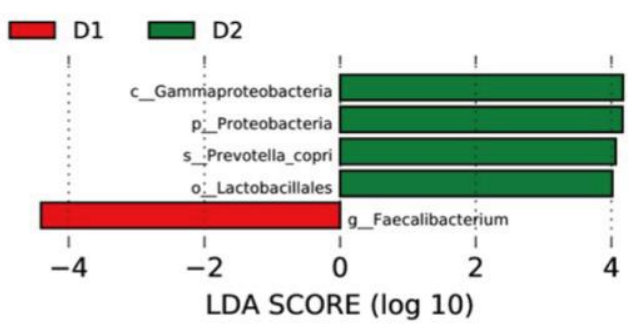

C

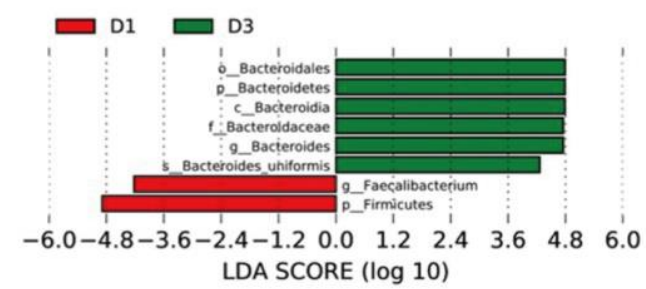

$E$

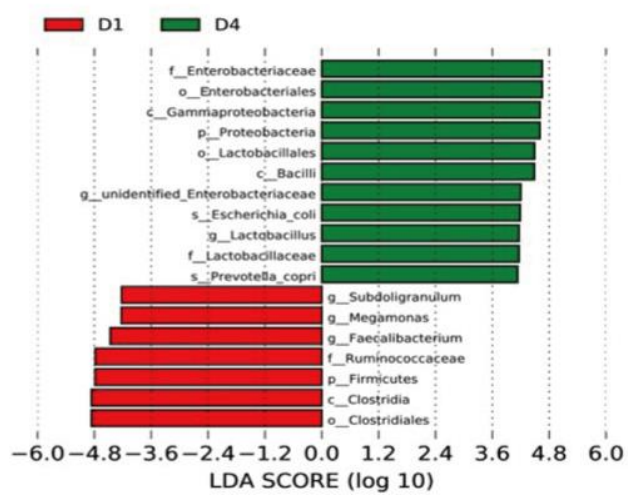

Cladogram

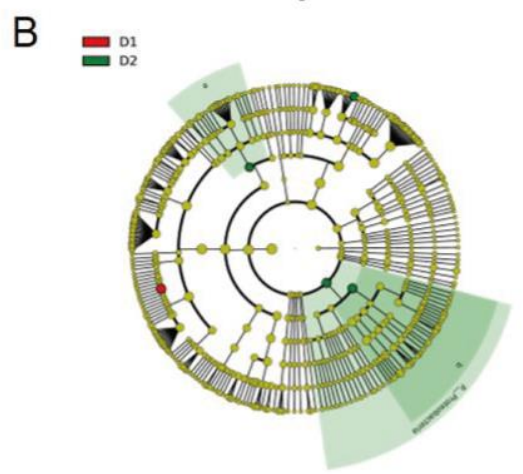

Cladogram

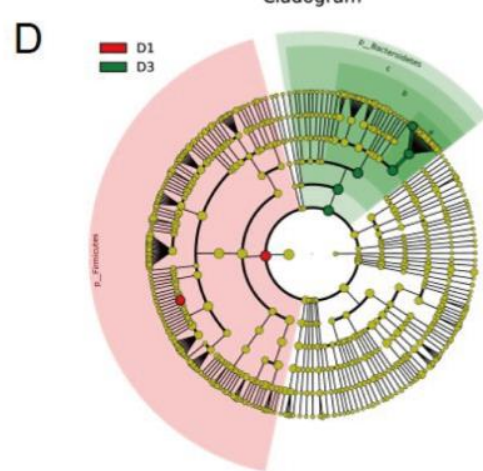

Cladogram

$\mathrm{F} \quad \mathrm{E}_{\mathrm{D4}}^{\mathrm{D}}$

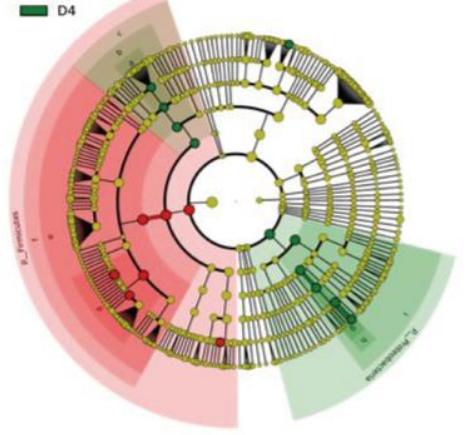

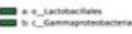

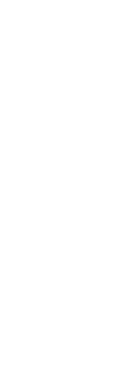

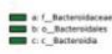

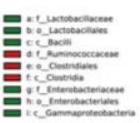

Figure 4: Different structures of gut microbiota in patients and healthy subjects

by LEfSe analysis. A,C,E: Specific species of gut microbiota between two groups by LEfSe analysis, the histogram shows the LDA scores, the lateral text shows the different species between two groups; B,D,F: LEfSe cladogram, showing the different species from phylum level (outer circle) to species level (inner circle), the red and green cladogram represent different groups, the abundance is proportional to the diameter of circle. A,B: D2 vs D1 groups; C,D: D3 vs D1 groups; E,F: D4 vs D1 groups. LDA SCORE=4. (D1: Healthy subjects; D2: Type 2 diabetes; D3: Cholelithiasis; D4: type 2 diabetes complicated with cholelithiasis.) 
A

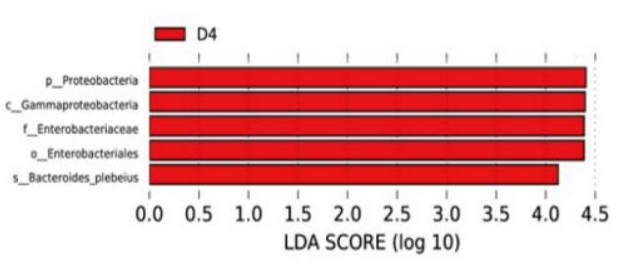

C

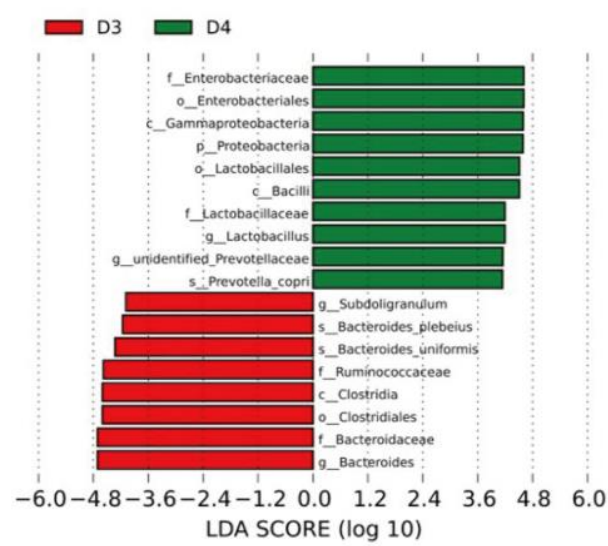

B

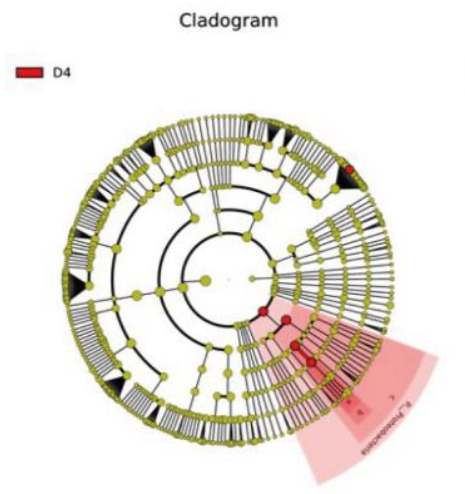

D

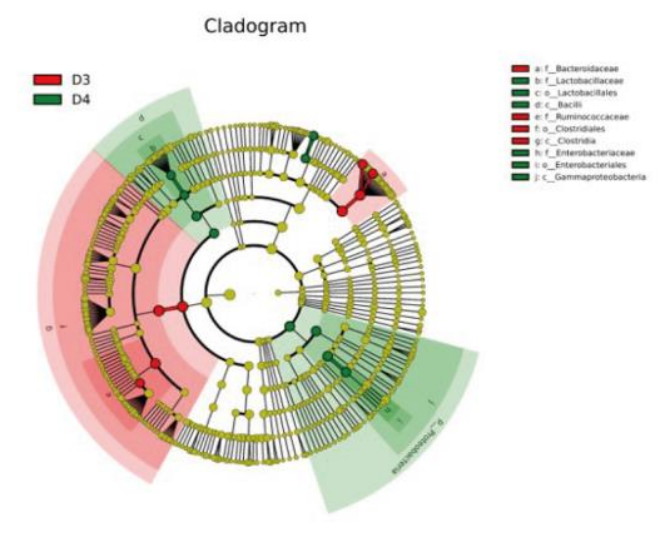

Figure 5: Different structures of gut microbiota among disease groups by LEfSe analysis. A,C: Specific species of gut microbiota between two groups by LEfSe analysis, the histogram shows the LDA scores, the lateral text shows the different species between two groups; B,D: LEfSe cladogram, showing the different species from phylum level (outer circle) to species level (inner circle), the red and green cladogram represent different groups, the abundance is proportional to the diameter of circle. A,B: D4 vs D2 groups; C,D: D4 vs D3 groups. LDA SCORE = 4. (D2: Type 2 diabetes; D3: Cholelithiasis; D4: type 2 diabetes complicated with cholelithiasis.) 
A

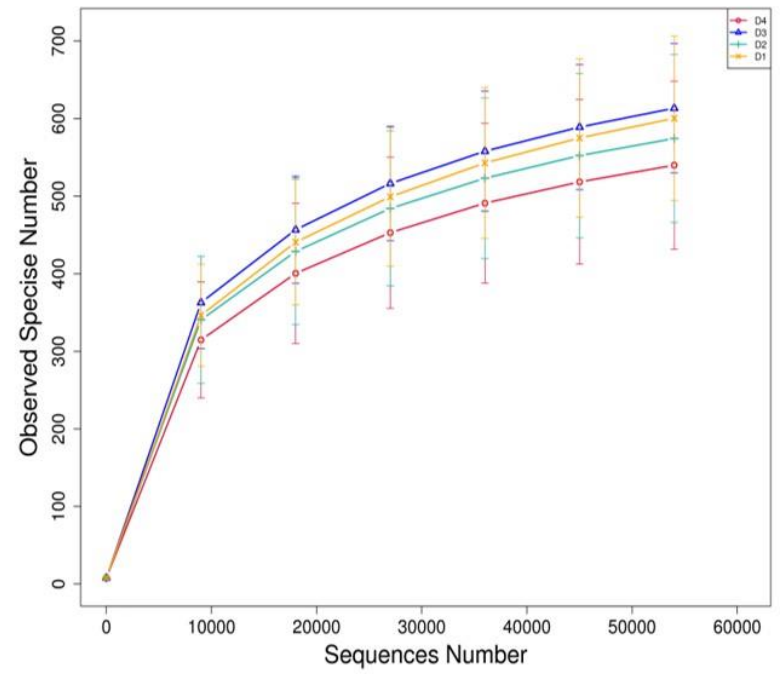

B

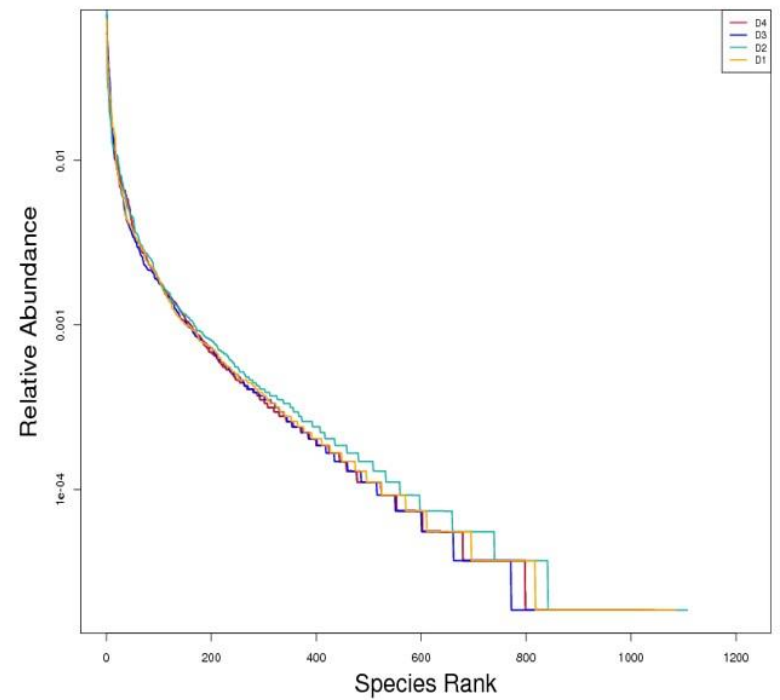

C

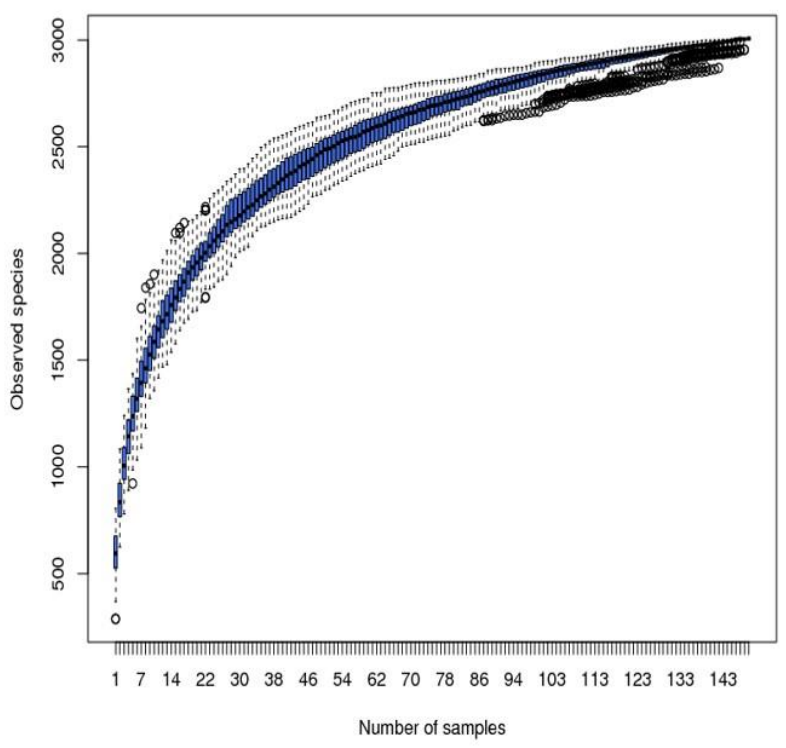

Fig S1 


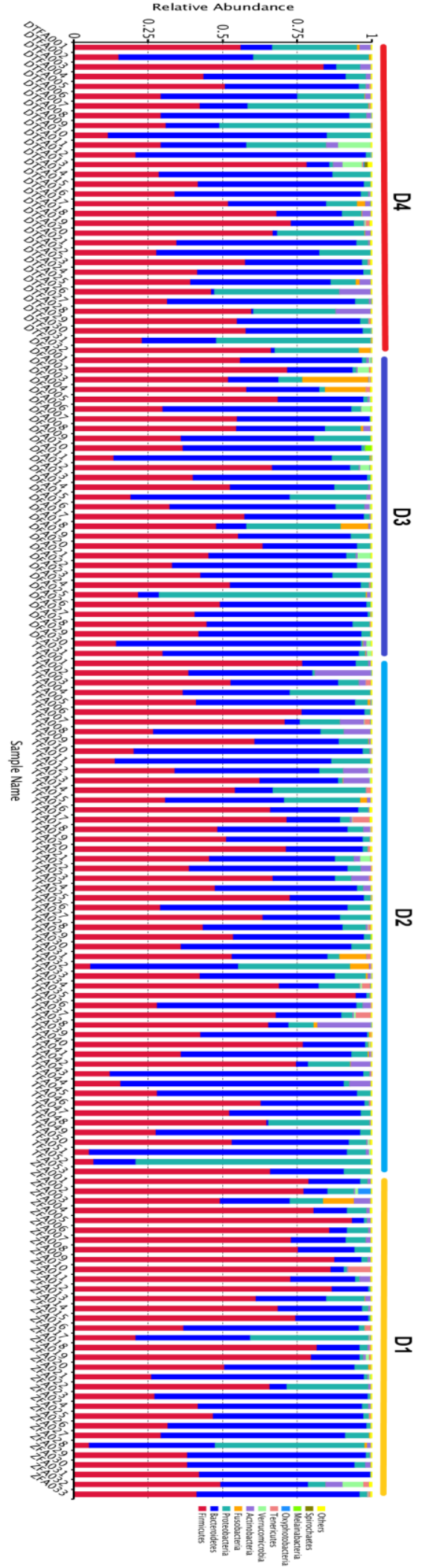

Fig S2

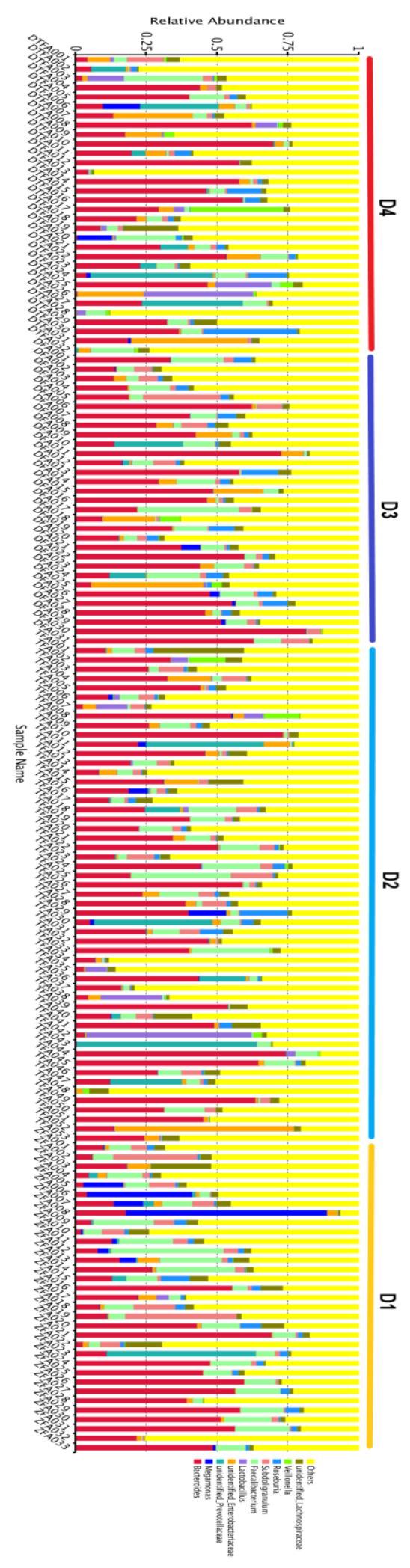

Fig S3 


\section{Figures}
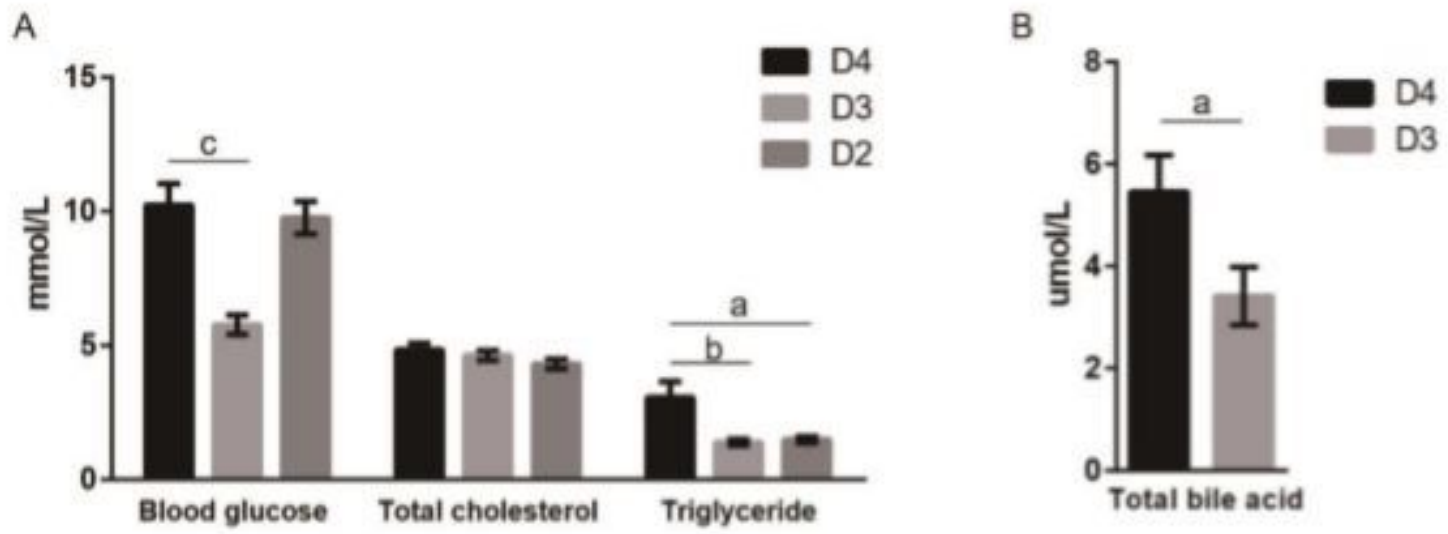

Figure 1

Serum metabolites levels. A: Blood glucose, total cholesterol and triglyceride levels in D2, D3 and D4 groups; B: Total bile acids levels in D3 and D4 groups. The levels were expressed as mean \pm SEM. (D2: Type 2 diabetes; D3: Cholelithiasis; D4: type 2 diabetes complicated with cholelithiasis. $a P<0.05, b P<0.01$, cP<0.001, Unpaired Student's t-test.)
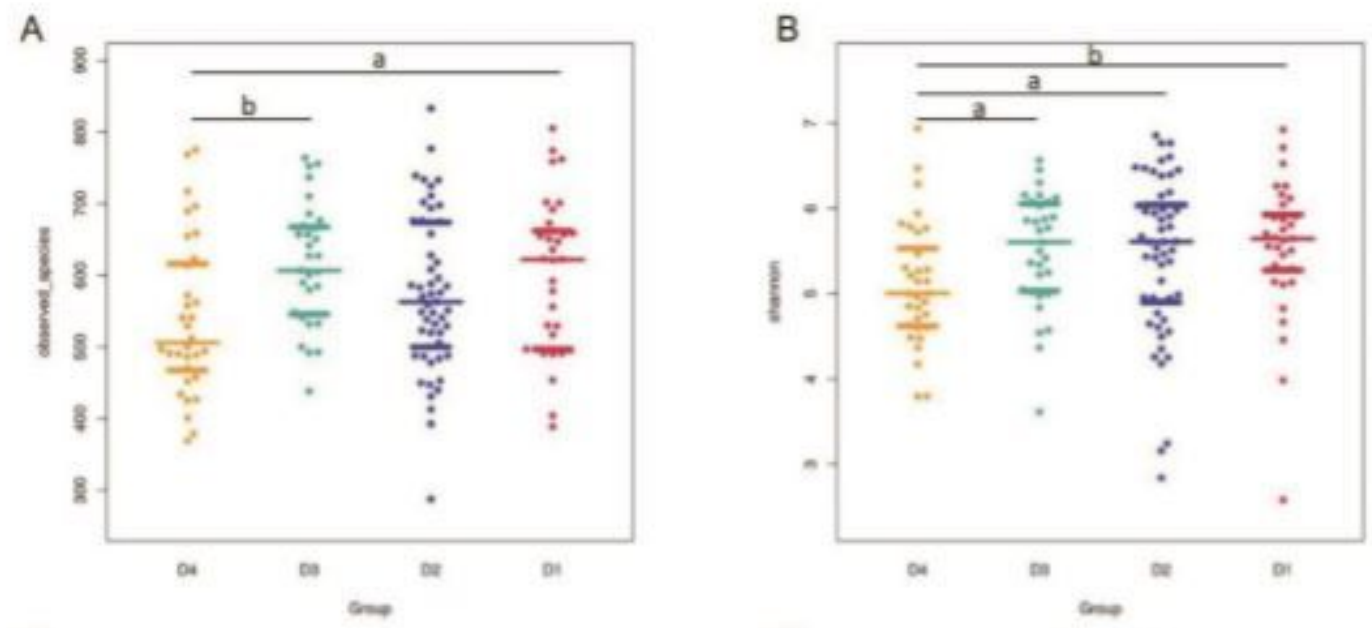

C

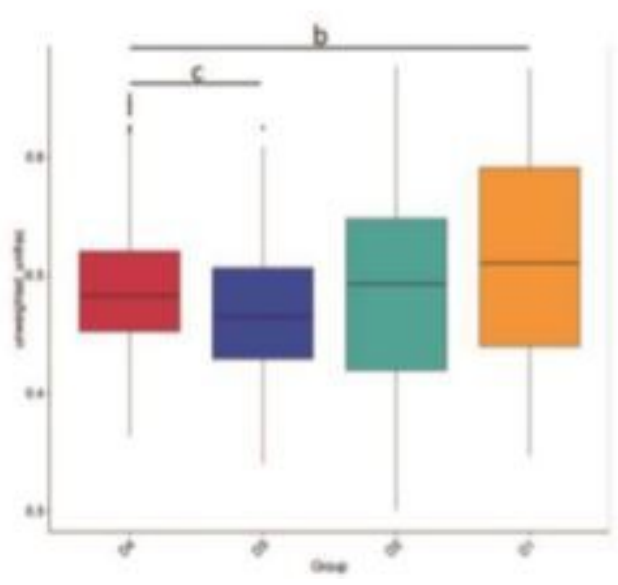

D

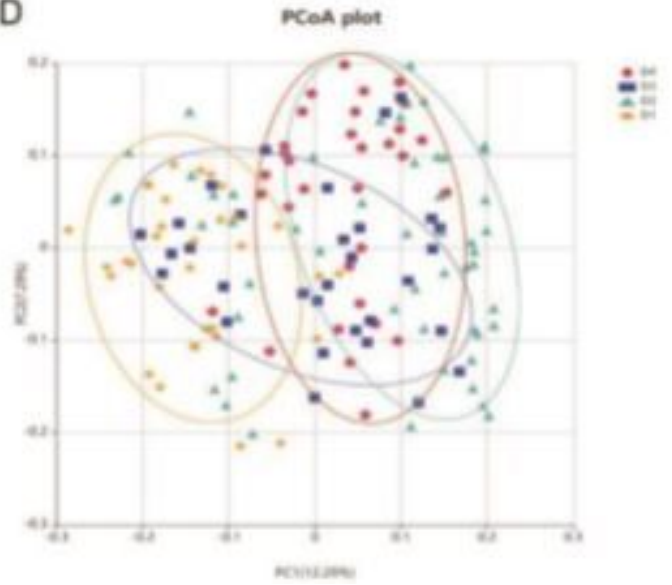




\section{Figure 2}

Gut microbial diversity analysis. A: Observed species, the scatter distribution of total number of species, namely the richness; B: Shannon index, namely species diversity and evenness; C: Unweighted unifrac based-beta diversity analysis, assessing gut microbial community differences; D: PCoA, showing the microbial community structure similarity. (D1: Healthy subjects; D2: Type 2 diabetes; D3: Cholelithiasis; D4: type 2 diabetes complicated with cholelithiasis. $\mathrm{aP}<0.05, \mathrm{bP}<0.01, \mathrm{CP}<0.001 ; \mathrm{A}, \mathrm{B}, \mathrm{C}$ : Wilcoxon ranksum test; D: AMOVA)
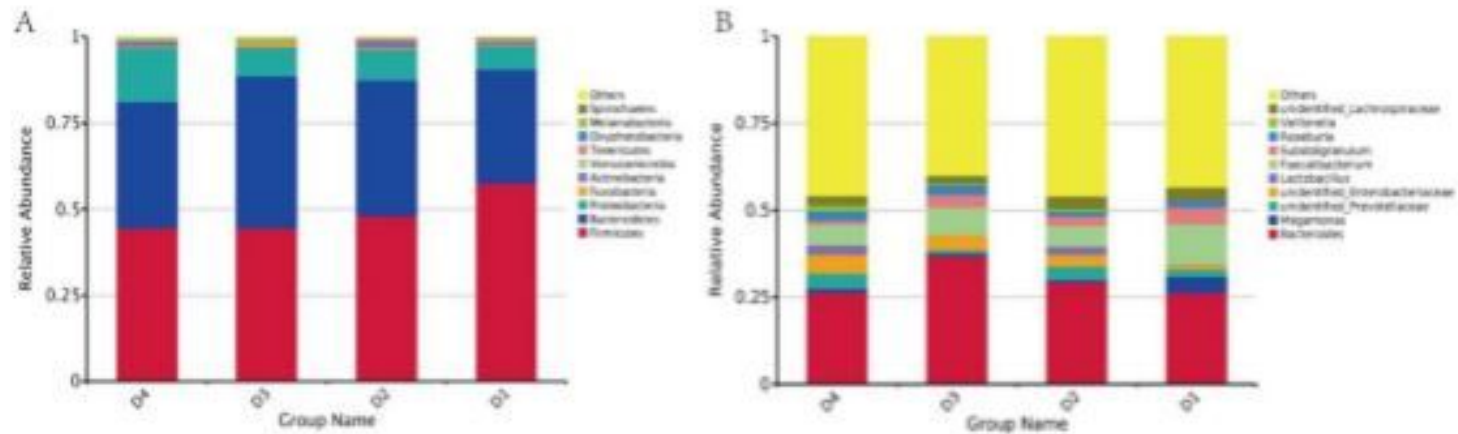

C
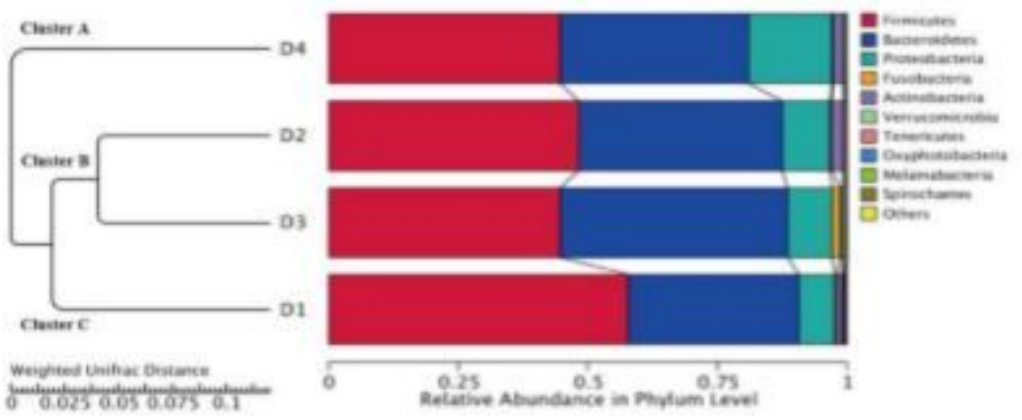

D

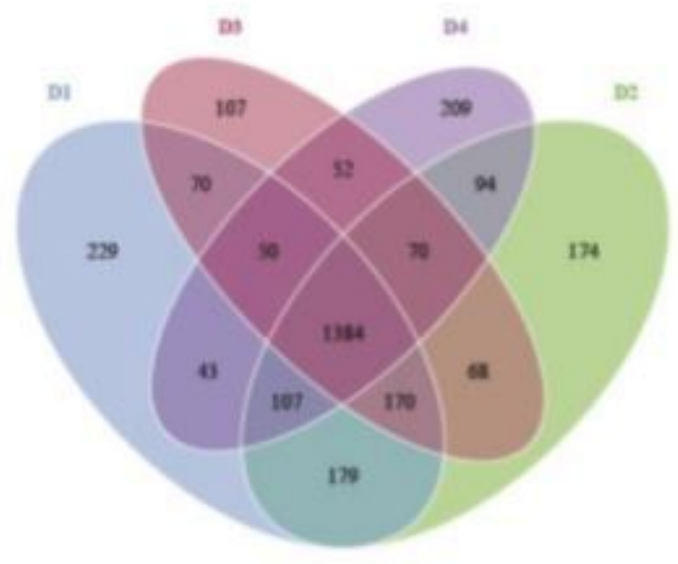

\section{Figure 3}

Gut microbial diversity analysis. A: Observed species, the scatter distribution of total number of species, namely the richness; B: Shannon index, namely species diversity and evenness; C: Unweighted unifrac based-beta diversity analysis, assessing gut microbial community differences; D: PCoA, showing the microbial community structure similarity. (D1: Healthy subjects; D2: Type 2 diabetes; D3: Cholelithiasis; 
D4: type 2 diabetes complicated with cholelithiasis. $\mathrm{aP}<0.05, \mathrm{bP}<0.01, \mathrm{cP}<0.001 ; \mathrm{A}, \mathrm{B}, \mathrm{C}$ : Wilcoxon ranksum test; D: AMOVA)

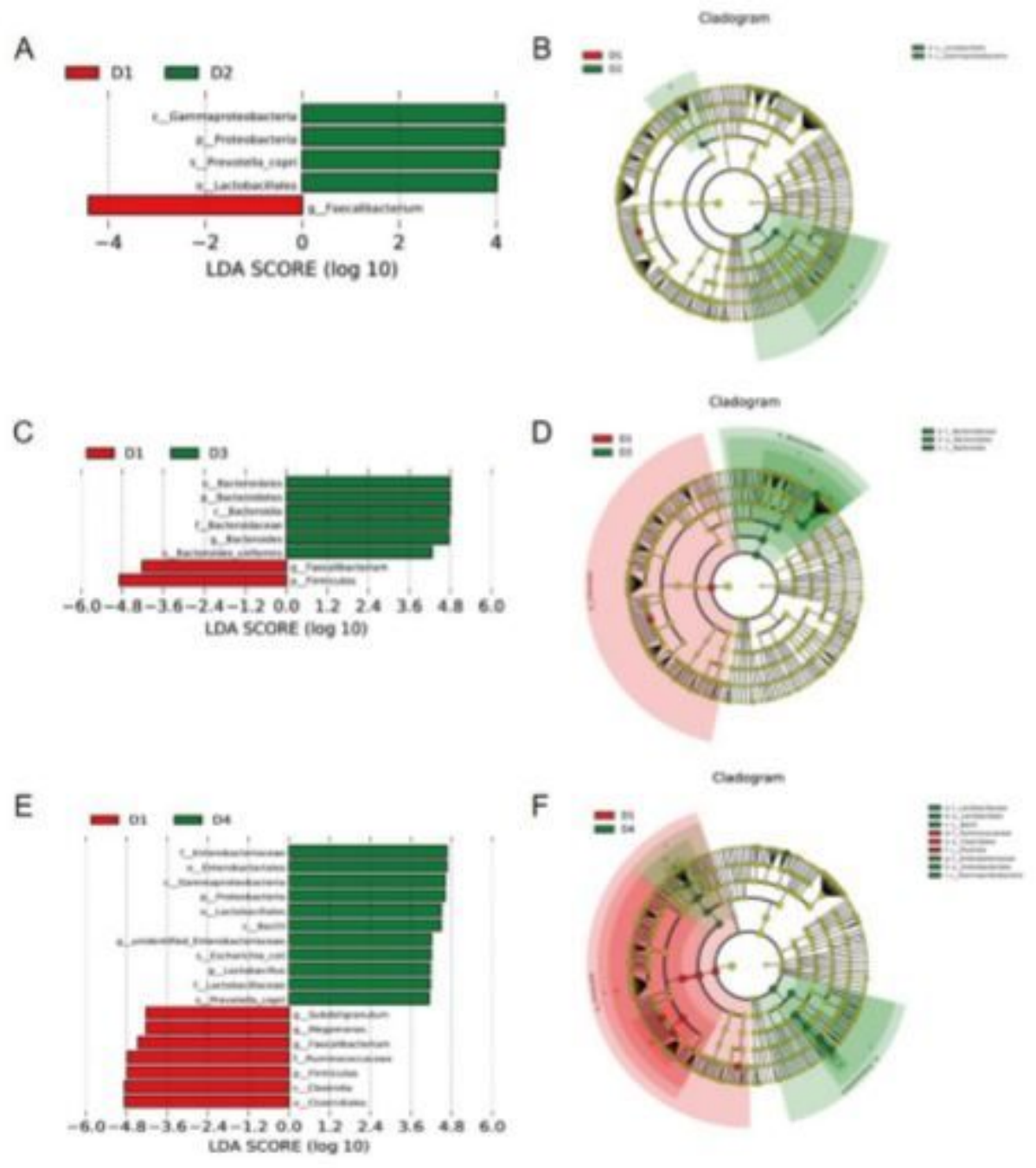

\section{Figure 4}

Different structures of gut microbiota in patients and healthy subjects by LEfSe analysis. A,C,E: Specific species of gut microbiota between two groups by LEfSe analysis, the histogram shows the LDA scores, the lateral text shows the different species between two groups; B,D,F: LEfSe cladogram, showing the different species from phylum level (outer circle) to species level (inner circle), the red and green cladogram represent different groups, the abundance is proportional to the diameter of circle. A,B: D2 vs D1 groups; C,D: D3 vs D1 groups; E,F: D4 vs D1 groups. LDA SCORE=4. (D1: Healthy subjects; D2: Type 2 diabetes; D3: Cholelithiasis; D4: type 2 diabetes complicated with cholelithiasis.) 
A

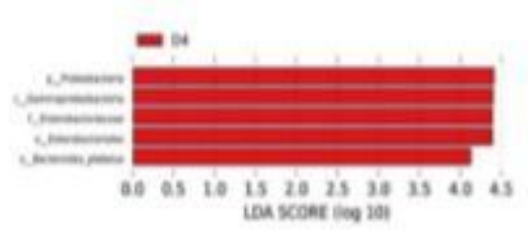

C

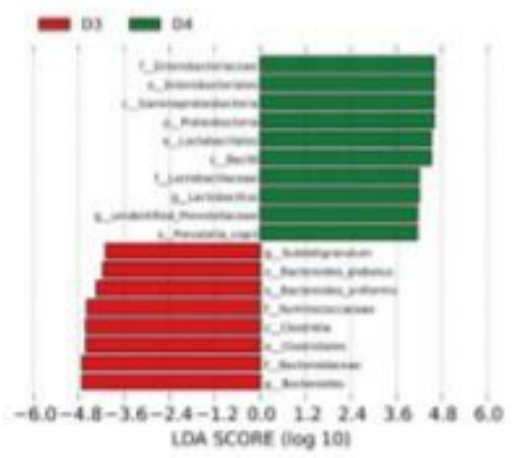

B

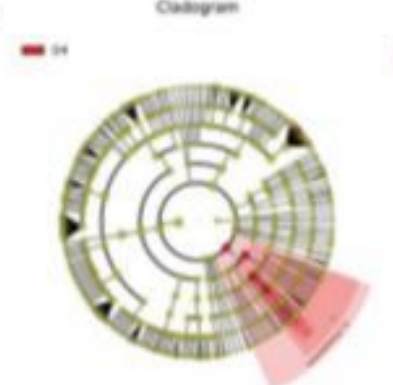

D

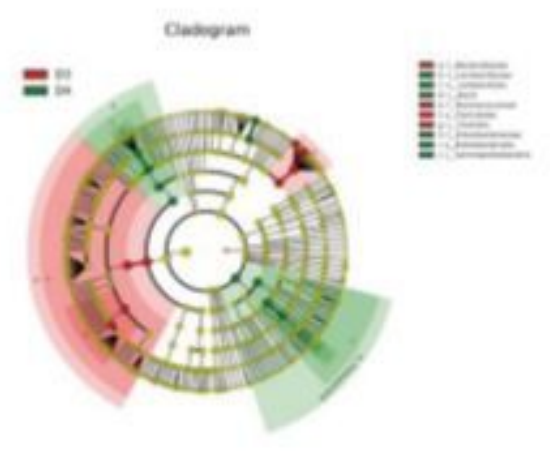

Figure 5

Different structures of gut microbiota among disease groups by LEfSe analysis. A,C: Specific species of gut microbiota between two groups by LEfSe analysis, the histogram shows the LDA scores, the lateral text shows the different species between two groups; B,D: LEfSe cladogram, showing the different species from phylum level (outer circle) to species level (inner circle), the red and green cladogram represent different groups, the abundance is proportional to the diameter of circle. A,B: D4 vs D2 groups; C,D: D4 vs D3 groups. LDA SCORE = 4. (D2: Type 2 diabetes; D3: Cholelithiasis; D4: type 2 diabetes complicated with cholelithiasis.) 